\section{Avaliação da efetividade da promoção da atividade física por agentes comunitários de saúde em visitas domiciliares}

\author{
Evaluation of the effectiveness of home-based \\ physical activity promotion by \\ community health workers
}

\author{
Evaluación de la efectividad de la promoción de la \\ actividad física por agentes comunitarios \\ de salud en visitas a domicilios
}

\begin{abstract}
This study analyzed the effectiveness of physical activity promotion by community health workers (CHW) during home visits. This was a non-randomized controlled trial that lasted six months, with one group of $\mathrm{CHW}$ that received training to promote physical activity during home visits among users of the Brazilian Unified National Health System (SUS) and a control group. Physical activity and stages of behavior change were evaluated in 176 adults $(n=90$ in the intervention group and $n=86$ in the control group) assisted by the CHW. Associations, prevalence ratios, and generalized estimate equations were conductec to verify differences between groups. No evidence of differences in physical activity and stages of behavior change were observed between the two groups. CHW from the intervention group conducted more home visits promoting physical activity among elders, those with low schooling, unemployed, and those with chronic diseases. It is important to reassess the work and priorities of CHW to expand physical activity promotion under the SUS.
\end{abstract}

Community Health Workers; Home Visit; Motor Activity

\author{
Evelyn Fabiana Costa 1,2 \\ Douglas Roque Andrade 2,3 \\ Leandro Martin Totaro Garcia 1,2 \\ Evelyn Helena Corgosinho Ribeiro 1,2 \\ Taynã Ishi dos Santos 1,2 \\ Alex Antonio Florindo 2,3
}

\section{Resumo}

Este estudo verificou a efetividade da promoção de atividade física realizada por agentes comunitários de saúde (ACS) em visitas domiciliares. Trata-se de um ensaio controlado não randomizado com duração de seis meses, com um grupo de ACS que passou por um processo educativo para promover atividade física nas visitas domiciliares para intervir em usuários do Sistema Único de Saúde (SUS) e um grupo controle. Foram avaliados a prática de atividade física e os estágios de mudança de comportamento em 176 adultos ( $n=90$ do grupo intervenção e $n=$ 86 do grupo controle) atendidos pelos ACS. Foram realizadas análises de associação, razões de prevalência e equações de estimativas generalizadas para verificar diferenças entre os grupos. Não foram observadas evidências de diferenças nos níveis de atividade física e nos estágios de mudança de comportamento entre os usuários dos dois grupos. Os ACS do grupo intervenção realizaram mais visitas domiciliares para promover atividade física aos idosos, pessoas com baixa escolaridade, que não trabalhavam e que tinham doenças crônicas. É importante que os modos de trabalho e prioridades dos ACS sejam repensados para ampliar a promoção da atividade física no SUS.

Agentes Comunitários de Saúde; Visita Domiciliar; Atividade Motora 


\section{Introdução}

A visita domiciliar, prática antiga no campo da saúde, é uma oportunidade diferente para o cuidado num espaço extraunidade, caracterizada por ser uma tecnologia leve 1 , dando vistas às condições culturais e socioeconômicas de cada família, indivíduo ou coletividade, que podem interferir no processo saúde-doença. Ceccim \& Machado 2 descrevem a visita domiciliar como promotora da ampliação de informações e conhecimento (autocuidado, recursos sociais e ações políticas), voltadas ao atendimento assistencial (diagnóstico e tratamento) ou educativo 3 . A valorização da Estratégia Saúde da Família (ESF) no Brasil como proposta de reorientação do modelo assistencial de Atenção Básica possui o domicílio como um dos elementos-chave do espaço de atuação ${ }^{4}$. A visita domiciliar é um dos instrumentos mais relevantes do trabalho dos profissionais da ESF, pois possibilita conhecer os problemas, fragilidades e necessidades de saúde dos usuários em seu domicílio, além de permitir que o profissional interaja com o meio em que os indivíduos vivem e de observar, conhecer e se aproximar da sua realidade.

De acordo com o Departamento de Gestão da Educação na Saúde do Ministério da Saúde, uma das cinco competências atribuídas aos agentes comunitários de saúde (ACS) é o desenvolvimento de ações em promoção da saúde por meio de atividades educativas 5 . Alguns estudos demonstraram que a visita domiciliar realizada por ACS é um instrumento efetivo para a execução de ações de prevenção de doenças e promoção da saúde, especialmente quando associadas a um processo de educação permanente, contribuindo para a melhora de indicadores de saúde da criança, controle de pré-natal e imunizações, vigilância em saúde, uso adequado de medicamentos e controle da dengue $6,7,8,9$.

No que diz respeito aos processos educativos, em vários países, cursos têm sido desenvolvidos com ACS para que eles promovam atividade física e alimentação saudável para a população com uso de estratégias como organização de grupos de prática de atividade física, encontros de educação em saúde e visita domiciliar 10,11,12,13. Nos Estados Unidos, o aumento na prática de atividade física foi encontrado como resultado de estudos de intervenções promovidas por ACS por meio das visitas às populações carentes 14,15.

Frente às demandas individuais e comunitárias, a promoção da atividade física realizada pelos ACS por meio das visitas domiciliares ainda é pouco utilizada nas unidades básicas de saúde (UBS) no Brasil. O único estudo encontrado na literatura que investigou o ACS como profissio- nal promotor de atividade física foi o de Gomes \& Duarte 16. Esses autores desenvolveram e avaliaram uma intervenção de aconselhamento de atividade física aos usuários da ESF de Florianópolis (Santa Catarina) e obtiveram resultados significativos para a promoção da atividade física. Na ocasião, os adultos receberam visita domiciliar do ACS acompanhado de um profissional de Educação Física.

A mudança do nível de atividade física populacional é complexa e não vem ocorrendo no Brasil, particularmente nas atividades praticadas nos momentos de lazer 17 em que $60 \%$ dos adultos brasileiros são inativos 18. O Ministério da Saúde, por meio do Sistema Único de Saúde (SUS), tem investido em ações de promoção da atividade física 19 e o cenário atual é promissor na opinião de médicos e enfermeiros que atuam na Atenção Básica que consideram importante promover atividade física nas UBS 20 . No entanto os ACS ainda são pouco envolvidos como atores importantes na promoção da atividade física no Brasil, apesar de seu potencial por possuírem características de atuação singulares e manterem contato frequente com as pessoas da comunidade, dada que a maior parte das suas ações é realizada extraunidade.

Dessa forma, foi realizado um processo de formação em educação profissional para que os ACS se tornassem promotores de atividade física nas visitas domiciliares 21 . Nesse sentido e com base nesse curso, o objetivo de tal estudo foi verificar a efetividade de uma intervenção para a promoção da atividade física por meio das visitas domiciliares promovidas por ACS aos usuários do SUS atendidos pela ESF.

\section{Métodos}

Trata-se de um ensaio controlado não randomizado que fez parte de uma pesquisa maior denominada Estudo de Intervenções para a Promoção da Atividade Física no SUS pela ESF, realizada entre os anos de 2011 e 2012, no distrito de Ermelino Matarazzo, extremo leste do Município de São Paulo 22. Todos os resultados estão sendo sintetizados e utilizados como um conjunto de ações de promoção da atividade física aplicadas na região e que foram denominadas de "Ambiente Ativo".

\section{Contexto da intervenção}

Foram escolhidas duas UBS, uma para intervenção e outra para o controle. A escolha intencional ocorreu porque as UBS faziam parte do contexto de outras intervenções da pesquisa maior. Entre- 
tanto, a similaridade entre as áreas de cobertura (tamanho e perfil da população adstrita) foi considerada para a escolha.

Em julho de 2011, a UBS-intervenção era composta por seis equipes de ESF e 6.007 famílias estavam cadastradas (20.960 pessoas), enquanto a UBS-controle era composta por sete equipes com 6.935 famílias cadastradas (24.366 pessoas). Ambas as UBS atuavam com equipes compostas por cinco ACS (todas mulheres).

A intervenção constou de um processo de formação em educação profissional para a promoção de atividade física no lazer e como forma de deslocamento por meio das visitas domiciliares ministrado para as ACS da UBS-intervenção. O curso foi realizado durante a jornada de trabalho, com início no final de abril de 2011, e teve duração de 12 horas, em quatro encontros semanais de três horas. Utilizou-se como referenciais teóricos o modelo ecológico para a promoção da atividade física no nível da comunidade 23, o modelo transteórico para mudança de comportamento aplicado à atividade física 24 e a Política Nacional de Educação Permanente 25. O processo ensinoaprendizagem foi orientado por uma perspectiva construcionista social, que compreende e incorpora o conhecimento pela elaboração em conjunto de diálogo e trocas de saberes entre as pessoas que compõem o grupo 26 .

Em média, 29 ACS participaram de cada um dos encontros e a taxa de frequência para os quatro encontros foi igual ou superior a $93 \% 21$. Durante o curso, as ACS foram orientadas a realizarem as visitas domiciliares tendo em conta a sua rotina de trabalho, ou seja, de acordo com as diretrizes do Ministério da Saúde, que recomenda que haja no mínimo uma visita mensal a cada domicílio da área de atuação do ACS, podendo haver variações em função do estado de saúde dos seus habitantes 27 .

As ACS do grupo controle somente responderam aos questionários de avaliação quantitativa de conhecimentos sobre atividade física e mantiveram sua rotina de trabalho.

\section{Amostragem dos usuários}

Para o cálculo do tamanho da amostra, foi utilizado o estudo de Gomes \& Duarte 16. Eles encontraram um aumento de $25 \%$ de indivíduos classificados no estágio de manutenção no grupo experimental, enquanto no grupo controle houve um decréscimo (10\%) na avaliação pós-intervenção. Adotando-se erros $\alpha=5 \%$ e $\beta=20 \%$, o tamanho da amostra foi calculado em 58 pessoas por grupo. Para proteger-se dos efeitos da não resposta, possíveis perdas e recusas, foram somados $50 \%$ ao cálculo amostral. Sendo assim, o tamanho da amostra foi calculado em 87 pessoas por grupo, totalizando 174 pessoas. O número inicial de 33 domicílios a serem sorteados para cada UBS foi pensado levando em conta que cada família teria entre dois e três adultos por casa.

A seleção dos participantes foi realizada por sorteio, considerando a quantidade de equipes e de famílias cadastradas em cada UBS, nas seguintes etapas: (1) ordenação dentro de cada equipe de todas as famílias cadastradas de forma crescente e numérica de acordo com o seu número de cadastro na UBS; (2) sorteio de 33 famílias em cada UBS. Nessa etapa, se a casa da família sorteada estivesse vaga ou se a família não existisse, era escolhida para substituí-la a família com numeração de cadastro imediatamente superior na UBS; (3) definição das casas vizinhas ao domicílio da família sorteada para aumentar a quantidade de famílias da abordagem inicial, prevendo-se recusas e domicílios fechados e também porque estudos epidemiológicos anteriores na região mostraram que o apoio social de amigos e vizinhos foi associado com a prática de atividade física 28 .

No total, foram abordadas 126 famílias em ambas UBS, representando por volta de $1 \%$ das famílias atendidas nos territórios adstritos.

Foram incluídos todos os moradores fixos das famílias com 18 anos ou mais de idade. Os critérios de exclusão foram: (a) ter algum problema que impedisse a pessoa de realizar atividade física como forma de deslocamento (caminhada ou bicicleta), ou alguma atividade física no tempo de lazer na semana anterior à abordagem (por exemplo, fraturas de membros inferiores, acidente vascular cerebral ou alguma outra doença incapacitante); (b) ter algum déficit cognitivo ou doença que impedisse a pessoa de responder sozinha ao questionário; (c) mulheres grávidas; (d) pessoas com intenção de mudança de endereço durante o período de intervenção (seis meses); (e) famílias que já participavam de outra intervenção da pesquisa maior.

\section{Descrição da coleta e das variáveis}

Os dados foram coletados por seis entrevistadores treinados durante seis horas na semana anterior ao início do trabalho de campo. Eles não participaram da intervenção (processo educativo), nem das visitas domiciliares para promoção da atividade física.

A coleta da linha de base dos usuários iniciou-se em março de 2011, um mês antes do processo educativo realizado com as ACS do grupo intervenção, e se estendeu até o início de junho do mesmo ano. Já a avaliação pós-intervenção ocorreu entre os meses de outubro e dezem- 
bro (após quatro meses do término do curso). Esse intervalo foi proposital a fim de aguardar que as ACS tivessem a oportunidade de realizar as visitas domiciliares para a promoção da atividade física.

Para a avaliação do nível de atividade física, foi usada a versão longa do International Physical Activity Questionnaire (IPAQ), módulos de lazer e deslocamento. As questões foram referentes ao tipo de atividade física realizada na última semana, à frequência semanal e ao tempo diário em atividades de caminhada, de intensidade moderada e vigorosa no lazer e para a caminhada ou uso de bicicleta como forma de deslocamento. Para as atividades de intensidade vigorosa, a duração foi multiplicada por dois 29 . A atividade física no momento de lazer foi categorizada como faz ( $\geq 10$ minutos por semana) ou não faz $(<10$ minutos por semana). A atividade física total (soma das atividade física no lazer e como forma de deslocamento) foi analisada de duas formas: (1) total em minutos por semana; (2) atingem ou não atingem a recomendação, pelo menos $150 \mathrm{mi}$ nutos por semana de atividade física. Esse questionário já foi validado em amostra de adultos residentes em Ermelino Matarazzo 30.

Para compor a avaliação dos estágios de mudança de comportamento para a prática de atividade física no lazer, foram acrescentadas duas questões ao final do bloco de atividade física referentes aos estágios de pré-contemplação (indivíduos que eram inativos no lazer e que não pretendiam se tornar ativos nos seis meses seguintes), contemplação (indivíduos inativos no lazer, mas que pretendiam se tornar ativos nos seis meses seguintes) e preparação (indivíduos inativos no lazer, mas que pretendiam se tornar ativos nos 30 dias seguintes). Os estágios de ação e manutenção foram avaliados com base nas questões do IPAQ de acordo com o bloco de atividade física no lazer (ação: indivíduos que realizavam pelo menos 150 minutos por semana de atividade física no lazer há menos de seis meses; e manutenção: indivíduos que praticavam pelo menos 150 minutos de atividade física no lazer há mais de seis meses) 24 .

No questionário da avaliação pós-intervenção foi incluído um bloco de questões referente ao histórico de visita domiciliar que era composto por cinco perguntas.

Foram utilizadas informações coletadas dos usuários acerca das características sociais, demográficas e de saúde como: sexo, idade em anos, situação conjugal, escolaridade em anos completos de estudo, trabalho, percepção de saúde e de qualidade de vida e relato de diagnóstico prévio realizado por médico de hipertensão arterial ou diabetes.

\section{Análise dos dados}

As variáveis foram descritas por meio de frequências, médias e desvios padrão (DP). As análises das variáveis de atividade física e estágios de mudança de comportamento foram realizadas de acordo com o princípio de intenção de tratar, no qual as respostas obtidas na linha de base foram repetidas na avaliação pós-intervenção para os que não foram reavaliados.

Para comparar as características sociodemográficas e as visitas domiciliares entre os grupos, utilizou-se o teste exato de Fisher e testes qui-quadrado com correção de Yates ou de tendência linear.

Em seguida, analisou-se por meio de regressão de Poisson a associação do relato dos usuários do recebimento da visita domiciliar das ACS para a promoção da atividade física com as seguintes variáveis: (a) sexo; (b) idade; (c) escolaridade; (d) status de trabalho; e (e) relato de hipertensão arterial ou diabetes. Os resultados foram apresentados em razões de prevalências (RP) e intervalo de 95\% de confiança (IC95\%).

Por fim, analisou-se o efeito da intervenção sobre os estágios de mudança de comportamento e dos níveis de atividade física no lazer e total (lazer e deslocamento) dos usuários por meio de equações de estimativas generalizadas. Para as variáveis de atividade física dicotômicas (faz ou não faz no lazer e atinge ou não atinge pelo menos 150 minutos por semana), utilizou-se função de distribuição binomial e função de ligação $l o$ git. Para os minutos semanais de atividade física, usou-se função de distribuição gaussiana inversa e função de ligação identidade. Neste caso, foi atribuído 1 minuto por semana a todos os valores para se evitar valores nulos. Para os estágios de mudança de comportamento, utilizou-se função de distribuição multinomial e função de ligação logit cumulativo. Em todos os casos, uma matriz de correlação não estruturada e o estimador Huber-White para a matriz de covariância foram empregados. Os modelos incluíram os efeitos principais de grupo (intervenção ou controle) e de momento de avaliação (tempo) e o efeito da interação entre ambos (tempo x grupo), além de ajuste para idade. Para as comparações múltiplas post hoc, foi utilizado o teste de Bonferroni.

Todos os dados foram analisados nos programas Stata, versão 12 (StataCorp LP, College Station, Estados Unidos), e IBM SPSS Statistics, versão 22.0 (IBM Corp., Armonk, Estados Unidos), adotando-se um valor de $\mathrm{p}<0,05$ para considerar as diferenças como significativas. 


\section{Aspectos éticos}

O projeto foi aprovado pelos Comitês de Ética da Secretaria Municipal de Saúde de São Paulo (protocolo CAEE 0072.0.162.000-10) e da Faculdade de Saúde Pública da Universidade de São Paulo (protocolo CAAE 03102212.0.0000.5421) e está registrado na base do Registro Brasileiro de Ensaios Clínicos e na base internacional ClinicalTrials.gov (identificador NCT01857648).

Todos os sujeitos foram esclarecidos acerca dos objetivos e procedimentos do estudo e àqueles que desejaram tomar parte assinaram um termo de consentimento livre e esclarecido. A intervenção apresentou riscos mínimos aos participantes e permitiu a saída do indivíduo a qualquer momento, sem prejuízo de seus cuidados.

As ACS do grupo controle receberam o mesmo processo educativo após o período da avaliação final da pesquisa. Isso foi feito de forma a garantir o mesmo tratamento para os usuários do SUS atendidos pelas ACS nas áreas do grupo controle.

\section{Resultados}

As informações referentes ao processo de seleção da amostra por domicílios/famílias e por moradores estão apresentadas na Figura 1. Visitaramse 126 domicílios/famílias, dos quais 292 adultos cadastrados pelas equipes da ESF foram abordados. A taxa de resposta da segunda avaliação foi de $89,8 \%$ e não foram encontradas diferenças quanto ao sexo $(\mathrm{p}=0,934)$, idade $(\mathrm{p}=0,064)$ e escolaridade $(p=0,356)$ entre os sujeitos que não participaram da avaliação pós-intervenção (perdas de seguimento) quando comparado aos que participaram.

A Tabela 1 apresenta a descrição da amostra total e estratificada por grupo. Com exceção da idade, as demais variáveis não apresentaram diferença significativa entre os grupos.

Acerca do recebimento das visitas domiciliares realizadas pelas ACS, somente a quantidade de visitas recebidas nos últimos seis meses apresentou diferença entre os grupos, pois os adultos cadastrados nas áreas de atuação das ACS do grupo intervenção receberam mais visitas do que os usuários das áreas do grupo controle. $\mathrm{Na}$ observação da amostra total, a maior parte referiu ter recebido visita das ACS nos últimos seis meses. Contudo, $44,3 \%$ dos usuários que entraram no grupo intervenção não receberam visitas. Somente $27 \%$ dos indivíduos participantes que referiram ter recebido a visita domiciliar da ACS nos últimos seis meses responderam que a ACS conversou acerca da prática de atividade física.
Das pessoas que receberam tal orientação, 96,3\% gostaram das informações, e $74,1 \%$ se sentiram estimuladas a praticar ou a se inserir num programa de atividade física (Tabela 2).

Na Tabela 3, são apresentadas as relações das características sociais e demográficas dos usuários com o relato de recebimento de visitas de promoção da atividade física realizadas pelas ACS. Foi possível verificar que as ACS do grupo intervenção falaram mais de atividade física aos idosos (RP = 3,18; IC95\%: 1,36-7,42), às pessoas com menor escolaridade $(\mathrm{RP}=2,74$; IC95\%: 1,66-7,51), aos que não trabalhavam $(\mathrm{RP}=3,61$; IC95\%: 1,32-9,85) e aos que relataram possuir diagnóstico médico de diabetes ou hipertensão (RP = 2,86; IC95\%: 1,21-6,73).

Os dados de atividade física e estágios de mudança de comportamento estão apresentados na Tabela 4. Observando os dados para a amostra total, menos de $26 \%$ relataram fazer alguma atividade física no lazer tanto na linha de base quanto na avaliação pós-intervenção. Entretanto, na avaliação da atividade física total, em torno de $40 \%$ da amostra atingiram a recomendação da prática de atividade física para a saúde nas duas avaliações. A maioria das pessoas encontrava-se no estágio pré-contemplativo para a prática de atividade física no lazer. Ao comparar os usuários do SUS dos grupos-controle e intervenção antes e após o processo educativo realizado com as ACS da área de intervenção, constatou-se que não aconteceram diferenças significativas tanto intragrupos quanto intergrupos para as variáveis de atividade física no lazer, atividade física total (lazer e deslocamento) e estágios de mudança de comportamento.

\section{Discussão}

Não foram encontradas diferenças significativas nos níveis de atividade física e nos estágios de mudança de comportamento para a prática de atividade física no lazer e total entre os grupos de usuários atendidos pelas ACS que foram submetidas ao processo de formação em educação profissional em comparação com o grupo controle. Observou-se que o grupo de ACS que recebeu o curso promoveu mais atividade física nas visitas domiciliares para a população idosa, pessoas com menor nível de escolaridade, que não trabalhavam e que possuíam o diagnóstico realizado pelo médico de hipertensão arterial ou diabetes.

No Brasil, Gomes \& Duarte 16 obtiveram resultados significativos quanto às mudanças nos estágios de comportamento para a atividade física (aumento de $25 \%$ dos adultos no estágio de manutenção) e aumento do escore de ati- 
Fluxograma do processo de seleção da amostra das famílias (etapa 1), dos moradores (etapa 2) e das avaliações pré- e pós-intervenção.

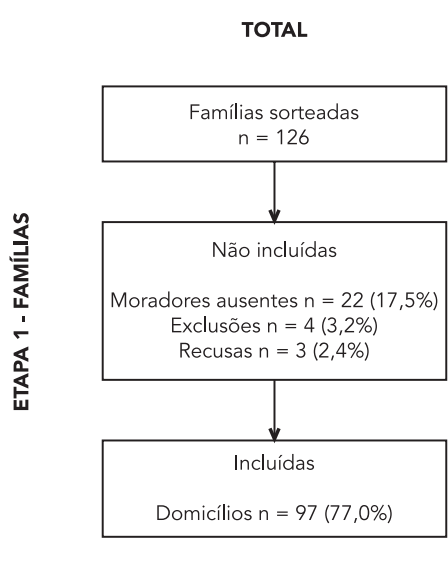

TOTAL

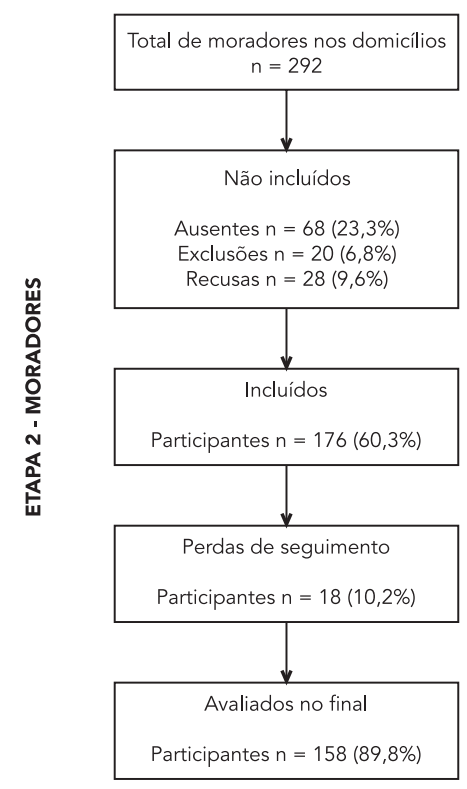

INTERVENÇÃO

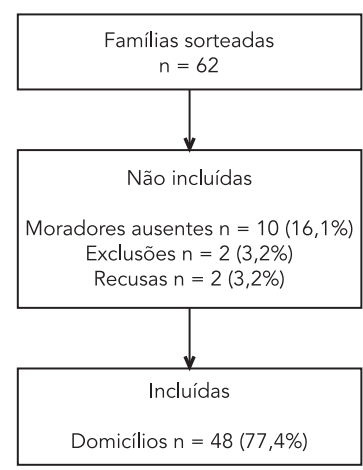

INTERVENÇÃO

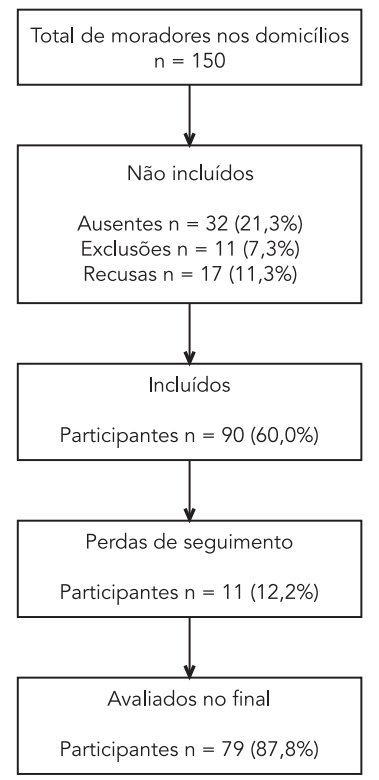

CONTROLE

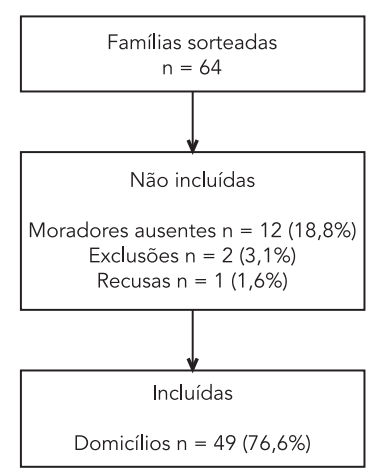

CONTROLE

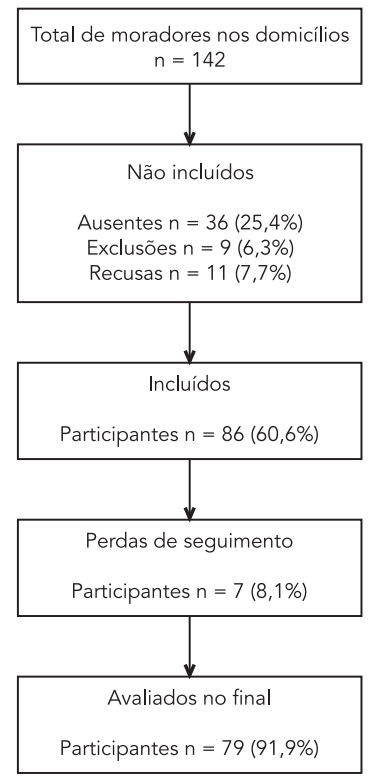

vidade física habitual de exercícios físicos. Porém, os profissionais de Educação Física foram os responsáveis pelas visitas domiciliares e pelas palestras educativas nas UBS ou em Centros Comunitários dos bairros, caracterizando uma combinação de estratégias realizada pelo especialista. Ademais, os autores utilizaram amostra intencional dos participantes, realizada pelas próprias ACS, e os dados na avaliação pré-intervenção foram coletados por elas, o que pode ter prejudicado as avaliações. Diferentemente, no presente estudo o processo educativo foi protagonizado pelos pesquisadores, mas as ACS não foram acompanhadas nas visitas domiciliares, proporcionando mais autonomia a elas. O processo de amostragem dos usuários analisados neste estudo foi aleatorizado e a coleta dos dados foi realizada por entrevistadores previamente treinados que não participaram do processo educativo. Além disso, as ACS não foram informadas sobre quais famílias foram sorteadas, o que pode ter representado melhor a realidade do 
Características sociais, demográficas e de saúde dos usuários das unidades de saúde intervenção e controle. Ermelino Matarazzo, Município de São Paulo, Brasil, 2011.

\begin{tabular}{|c|c|c|c|c|c|c|c|}
\hline \multirow[t]{2}{*}{ Variáveis } & \multicolumn{2}{|c|}{ Total } & \multicolumn{2}{|c|}{ Intervenção } & \multicolumn{2}{|c|}{ Controle } & \multirow[t]{2}{*}{ Valor de $p$ * } \\
\hline & n & $\%$ & $\mathrm{n}$ & $\%$ & $\mathrm{n}$ & $\%$ & \\
\hline \multicolumn{8}{|l|}{ Sexo } \\
\hline Homem & 73 & 41,5 & 37 & 41,1 & 36 & 41,9 & 1,000 \\
\hline Mulher & 103 & 58,5 & 53 & 58,9 & 50 & 58,1 & \\
\hline \multicolumn{8}{|l|}{ Idade (anos) } \\
\hline 18-39 & 80 & 45,5 & 36 & 40,0 & 44 & 51,2 & 0,040 \\
\hline $40-59$ & 67 & 38,1 & 34 & 37,8 & 33 & 38,4 & \\
\hline 60 ou mais & 29 & 16,4 & 20 & 22,2 & 9 & 10,4 & \\
\hline \multicolumn{8}{|l|}{ Estado marital } \\
\hline Casado ou com companheiro & 98 & 55,7 & 53 & 58,9 & 45 & 52,3 & 0,469 \\
\hline Sem companheiro & 78 & 44,3 & 37 & 41,1 & 41 & 47,7 & \\
\hline \multicolumn{8}{|l|}{ Escolaridade (anos completos) } \\
\hline $0-8$ & 89 & 50,6 & 40 & 44,4 & 49 & 57,0 & 0,131 \\
\hline 9 ou mais & 87 & 49,4 & 50 & 55,6 & 37 & 43,0 & \\
\hline \multicolumn{8}{|l|}{ Trabalho } \\
\hline Sim & 111 & 63,1 & 52 & 57,8 & 59 & 68,6 & 0,183 \\
\hline Não & 65 & 36,9 & 38 & 42,2 & 27 & 31,4 & \\
\hline \multicolumn{8}{|c|}{ Relato de diabetes ou hipertensão arterial } \\
\hline Sim & 50 & 28,4 & 27 & 30,0 & 23 & 26,7 & 0,755 \\
\hline Não & 126 & 71,6 & 63 & 70,0 & 63 & 73,3 & \\
\hline \multicolumn{8}{|l|}{ Tabagismo } \\
\hline Sim & 26 & 14,8 & 11 & 12,2 & 15 & 17,4 & 0,445 \\
\hline Não & 150 & 85,2 & 79 & 87,8 & 71 & 82,6 & \\
\hline \multicolumn{8}{|l|}{ Qualidade de vida } \\
\hline Ótima ou boa & 105 & 59,7 & 53 & 58,9 & 52 & 60,5 & 0,953 \\
\hline Regular, ruim ou muito ruim & 71 & 40,3 & 37 & 41,1 & 34 & 39,5 & \\
\hline \multicolumn{8}{|l|}{ Percepção de saúde } \\
\hline Ótima ou boa & 114 & 64,8 & 57 & 63,3 & 57 & 66,3 & 0,802 \\
\hline Regular, ruim ou muito ruim & 62 & 35,2 & 33 & 36,7 & 29 & 33,7 & \\
\hline
\end{tabular}

* Valor de p do teste qui-quadrado com correção de Yates ou de tendência linear.

trabalho dessas profissionais para verificar possíveis efeitos da intervenção.

Corroborando os resultados encontrados no presente estudo, Duggan et al. 31 não encontraram diferenças significativas para a mudança no nível de atividade física no lazer em adultos latinos residentes na cidade de Yakima (Washington, Estados Unidos) submetidos a cinco visitas domiciliares temáticas semanalmente, com duração de uma hora, realizadas por ACS após um treinamento de 100 horas. Por outro lado, resultados de estudo realizado com adultos indianos moradores da cidade de Nova Iorque (Estados Unidos), que receberam seis sessões mensais de visitas domiciliares para a promoção de atividade física de promotores de saúde, demonstraram que, após o período de intervenção, a proporção de participantes que referiram praticar atividade física na semana passou de $52 \%$ para $89 \% 15$.

É importante ressaltar que os ACS têm sido amplamente utilizados em intervenções de prevenção de doenças crônicas ou cuidados em saúde para populações carentes que residem nos Estados Unidos, sendo considerado um profissional potencialmente eficaz para melhorar o acesso aos cuidados de saúde 32 . Em algumas intervenções, a promoção da atividade física por meio das visitas domiciliares tem sido utilizada, mas, geralmente, os estudos utilizam encontros de educação em saúde no formato de palestras visando à promoção da atividade física e alimentação saudável e, em alguns casos, grupos de ca- 
Tabela 2

Descrição das visitas domiciliares realizadas pelas agentes comunitárias de saúde (ACS) por unidade de saúde. Ermelino Matarazzo, Município de São Paulo, Brasil, 2011.

\begin{tabular}{|c|c|c|c|c|c|c|c|}
\hline \multirow[t]{2}{*}{ Variáveis } & \multicolumn{2}{|c|}{ Total } & \multicolumn{2}{|c|}{ Intervenção } & \multicolumn{2}{|c|}{ Controle } & \multirow{2}{*}{$\begin{array}{c}\text { Valor de } \\
p^{*}\end{array}$} \\
\hline & n & $\%$ & $\mathbf{n}$ & $\%$ & n & $\%$ & \\
\hline \multicolumn{8}{|c|}{ Você recebeu visita domiciliar da ACS nos últimos seis meses? } \\
\hline Sim & 100 & 63,3 & 44 & 55,7 & 56 & 70,9 & 0,069 \\
\hline Não & 58 & 36,7 & 35 & 44,3 & 23 & 29,1 & \\
\hline \multicolumn{8}{|c|}{ Quantas visitas domiciliares você recebeu nesse período? ** } \\
\hline Menos de seis & 58 & 58,0 & 13 & 29,5 & 45 & 80,4 & $<0,001$ \\
\hline Seis ou mais & 42 & 42,0 & 31 & 70,5 & 11 & 19,6 & \\
\hline \multicolumn{8}{|c|}{ A ACS conversou com você sobre atividade física ou esporte em alguma visita } \\
\hline \multicolumn{8}{|c|}{ domiciliar nesse período? ** } \\
\hline $\operatorname{Sim}$ & 27 & 27,0 & 14 & 31,8 & 13 & 23,2 & 0,462 \\
\hline Não & 73 & 73,0 & 30 & 68,2 & 43 & 76,8 & \\
\hline \multicolumn{8}{|c|}{ Você gostou de receber informações acerca de atividade física oferecidas pela ACS? } \\
\hline \multicolumn{8}{|c|}{ 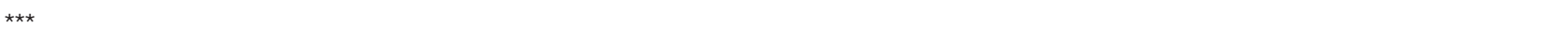 } \\
\hline Sim & 26 & 96,3 & 14 & 100,0 & 12 & 92,3 & 0,480 \\
\hline Não & 1 & 3,7 & - & - & 1 & 7,7 & \\
\hline \multicolumn{8}{|c|}{ A orientação estimulou você a praticar atividade física? *** } \\
\hline Sim & 20 & 74,1 & 11 & 78,6 & 9 & 69,2 & 0,680 \\
\hline Não & 7 & 25,9 & 3 & 21,4 & 4 & 30,8 & \\
\hline \multicolumn{8}{|c|}{ Anteriormente aos últimos seis meses, você recebeu visita domiciliar com } \\
\hline \multicolumn{8}{|c|}{ informações ou convites para grupos? } \\
\hline Sim & 17 & 10,8 & 11 & 13,9 & 6 & 7,6 & 0,304 \\
\hline Não & 141 & 89,2 & 68 & 86,1 & 73 & 92,4 & \\
\hline
\end{tabular}

* Valor de $\mathrm{p}$ do teste qui-quadrado com correção de Yates ou exato de Fisher;

** $\mathrm{n}$ total $=100$ (referente aos adultos que responderam ter recebido a visita domiciliar da ACS nos últimos seis meses);

$\star \star \star * n$ total $=27$ (referente aos adultos que confirmaram ter recebido a visita domiciliar da ACS para a promoção da atividade física).

minhada ou ginástica são oferecidos em espaços das comunidades como escolas, centros de saúde ou parques. Essas estratégias são oferecidas isoladamente ou combinadas 33,34 . Vale ressaltar que o sistema de saúde norte-americano e, obviamente, muitas das funções e atribuições dos ACS são diferentes em comparação com os ACS do Brasil.

As dificuldades encontradas pelas ACS para promover atividade física nas visitas domiciliares podem ser caracterizadas por limitações institucionais, como a sobrecarga de trabalho, a falta de reconhecimento e valorização, a dificuldade do trabalho em equipe e o desconhecimento das funções 35 . Isso pode ser uma das explicações do porquê não se conseguiu modificar o nível de atividade física dos usuários que receberam as visitas domiciliares das ACS que participaram do curso. Além disso, existem múltiplos fatores associados ou determinantes da prática de atividade física. Dessa forma, deve-se considerar, para além das questões relacionadas ao pro- cesso de trabalho dos ACS, fatores individuais, interpessoais e ambientais que interferem na mudança de comportamento para a prática de atividade física 36 .

Não é fácil realizar a visita domiciliar com total efetividade, uma vez que parte do tempo dos ACS é dedicada às atividades administrativas que descaracterizam sua função e, muitas vezes, o horário de trabalho não está adequado à realidade local 37 . Talvez, pela falta de uma clara delimitação das atribuições dos ACS, qualquer ação a ser desenvolvida no território seja atribuída a ele 38 , levando esses profissionais a desenvolver ações que nem sempre são de suas competências 39 .

Outra questão importante é o fato de que os ACS enfrentam problemas de cunho social ao adentrarem nos domicílios, o que pode levá-los a acreditar que falar da relação entre atividade física e saúde não seja pertinente e nem prioridade para o momento. Relações de gênero, violência doméstica, conflitos familiares, pessoas doentes, 
Recebimento das visitas domiciliares realizadas pelas agentes comunitárias de saúde (ACS), por unidade de saúde, para promover a prática de atividades físicas segundo sexo, idade, escolaridade, trabalho e presença de doenças crônicas. Ermelino Matarazzo, Município de São Paulo, Brasil, 2011.

\begin{tabular}{|c|c|c|c|c|c|c|c|}
\hline \multirow[t]{3}{*}{ Variáveis } & \multicolumn{7}{|c|}{ ACS conversou sobre atividade física nos últimos seis meses nas visitas domiciliares } \\
\hline & \multicolumn{3}{|c|}{ Intervenção } & \multicolumn{3}{|c|}{ Controle } & \multirow[t]{2}{*}{ Valor de $p$ ** } \\
\hline & n (\%) & RP (IC95\%) & Valor de $p$ * & $n(\%)$ & RP (IC95\%) & Valor de $p$ * & \\
\hline \multicolumn{8}{|l|}{ Sexo } \\
\hline Homem & $5(29,4)$ & 1,00 & 0,790 & $3(15,8)$ & 1,00 & 0,370 & 0,434 \\
\hline Mulher & $9(33,3)$ & $1,13(0,45-2,84)$ & & $10(27,0)$ & $1,71(0,53-5,55)$ & & 0,782 \\
\hline \multicolumn{8}{|l|}{ Idade (anos) } \\
\hline $18-59$ & $6(19,4)$ & 1,00 & 0,007 & $8(16,7)$ & 1,00 & 0,002 & 0,771 \\
\hline 60 ou mais & $8(61,5)$ & $3,18(1,36-7,42)$ & & $5(62,5)$ & $3,75(1,62-8,66)$ & & 0,965 \\
\hline \multicolumn{8}{|c|}{ Escolaridade (anos) } \\
\hline 9 ou mais & $4(17,4)$ & 1,00 & 0,046 & $1(5,6)$ & 1,00 & 0,085 & 0,363 \\
\hline $0-8$ & $10(47,6)$ & $2,74(1,66-7,51)$ & & $12(31,6)$ & $5,68(0,79-41,13)$ & & 0,268 \\
\hline \multicolumn{8}{|l|}{ Trabalha } \\
\hline Sim & $4(15,4)$ & 1,00 & 0,012 & $8(20,5)$ & 1,00 & 0,467 & 0,749 \\
\hline Não & $10(55,6)$ & $3,61(1,32-9,85)$ & & $5(29,4)$ & $1,43(0,54-3,78)$ & & 0,176 \\
\hline \multirow{2}{*}{\multicolumn{8}{|c|}{$\begin{array}{l}\text { Diabetes e/ou hipertensão } \\
\text { arterial }\end{array}$}} \\
\hline & & & & & & & \\
\hline Não & $6(20,0)$ & 1,00 & 0,016 & $6(18,2)$ & 1,00 & 0,293 & 0,854 \\
\hline Sim & $8(57,1)$ & $2,86(1,21-6,73)$ & & $7(30,4)$ & $1,67(0,64-4,37)$ & & 0,169 \\
\hline
\end{tabular}

IC95\%: intervalo de 95\% de confiança; RP: razão de prevalência.

* Valor de p da RP;

** Valor de $p$ do teste qui-quadrado com correção de Yates ou teste exato de Fisher, referente à associação entre o desfecho e os grupos, para cada nível das variáveis sexo, idade, escolaridade, status de trabalho e diabetes ou hipertensão arterial.

desemprego, abordagens com os adolescentes, número elevado de pessoas a serem acompanhadas e frustrações pelo fato de perceberem que seu trabalho não tem a efetividade que gostariam são situações com que os ACS relatam ter dificuldades de lidar 40,41 .

Ainda que o próprio ACS identifique a visita domiciliar como a atividade mais importante do seu trabalho e mais efetiva para realizar as orientações de educação e promoção da saúde ${ }^{42}$, verificou-se que as ações desses profissionais continuam reproduzindo o modelo de cuidado biomédico em detrimento à promoção da saúde ao priorizarem o tema atividade física na visita domiciliar para os adultos portadores de hipertensão ou diabetes. Ainda é forte a concepção biomédica do trabalho em saúde, privilegiandose o tratamento e a cura, em que grande parte dos trabalhadores, incluindo os ACS, não valoriza o potencial das ações de promoção da saúde 43 . Para além das dificuldades encontradas pelos ACS, os demais profissionais das equipes de saúde, especialmente na Atenção Básica, também encontram dificuldades para desenvolver ações de promoção em comunidades com caracterís- ticas tão singulares. Há uma tensão entre o que a população apresenta como demanda e o que os serviços oferecem. Muitas vezes, os profissionais têm por atribuição a identificação de necessidades que enfatizem os indicadores de doenças e não de promoção da saúde. Logo, os ACS tenderão a identificar e agir com os problemas de saúde apontados pela equipe e pelos programas oficiais como prioritários 44 .

Apesar disso, entende-se que a formação dos ACS para a promoção da atividade física e os cursos de educação permanente ou de capacitação são promissores 10,11,12,13,45. Nessa perspectiva, o processo de formação em educação profissional realizado com as ACS do distrito de Ermelino Matarazzo, apesar de não ter sido efetivo para aumentar o nível de atividade física e melhorar os estágios de mudança de comportamento da população atendida, proporcionou melhora no conhecimento das ACS acerca das recomendações de atividade física para a saúde, além da (re) significação acerca da prática de atividade física na vida pessoal e profissional, valorizando-se a possibilidade do autocuidado, transformando um conhecimento anterior mais funcionalista 
Tabela 4

Comparação pré- e pós-intervenção dos estágios de mudança de comportamento e da atividade física. Ermelino Matarazzo, Município de São Paulo, Brasil, 2011.

\begin{tabular}{|c|c|c|c|c|c|c|}
\hline \multirow[t]{2}{*}{ Variáveis } & \multicolumn{2}{|c|}{ Pré } & \multicolumn{2}{|c|}{ Pós } & \multicolumn{2}{|c|}{ Valor de p } \\
\hline & $\begin{array}{l}\text { Intervenção } \\
\text { n (\%) }\end{array}$ & $\begin{array}{l}\text { Controle } \\
\text { n (\%) }\end{array}$ & $\begin{array}{l}\text { Intervenção } \\
\text { n (\%) }\end{array}$ & $\begin{array}{l}\text { Controle } \\
\text { n (\%) }\end{array}$ & Tempo & Tempo $\mathrm{x}$ grupo \\
\hline \multicolumn{7}{|l|}{ Estágios de mudança de comportamento } \\
\hline Pré-contemplação & $36(40,0)$ & $38(44,2)$ & $42(46,7)$ & $40(46,5)$ & 0,481 & 0,733 \\
\hline Contemplação & $16(17,8)$ & $13(15,1)$ & $15(16,7)$ & $10(11,6)$ & & \\
\hline Preparação & $23(25,6)$ & $22(25,6)$ & $24(26,7)$ & $20(23,3)$ & & \\
\hline Ação & $4(4,4)$ & $5(5,8)$ & $2(2,2)$ & $8(9,3)$ & & \\
\hline Manutenção & $11(12,2)$ & $8(9,3)$ & $7(7,8)$ & $8(9,3)$ & & \\
\hline \multicolumn{7}{|l|}{ Atividade física no lazer } \\
\hline Não faz (< 10 minutos/semana) & $67(74,4)$ & $64(74,4)$ & $71(78,9)$ & $61(70,9)$ & 0,837 & 0,206 \\
\hline Faz ( $\geq 10$ minutos/semana) & $23(25,6)$ & $22(25,6)$ & $19(21,1)$ & $25(29,1)$ & & \\
\hline \multicolumn{7}{|l|}{ Atividade física total } \\
\hline $\begin{array}{l}\text { Não atinge recomendação (< } 150 \text { minutos/ } \\
\text { semana) }\end{array}$ & $47(52,2)$ & $48(55,8)$ & $48(53,3)$ & $49(57,0)$ & 0,769 & 0,988 \\
\hline \multirow[t]{2}{*}{$\begin{array}{l}\text { Atinge recomendação ( } \geq 150 \text { minutos/ } \\
\text { semana) }\end{array}$} & $43(47,8)$ & $38(44,2)$ & $42(46,7)$ & $37(43,0)$ & & \\
\hline & Média (DP) & Média (DP) & Média (DP) & Média (DP) & & \\
\hline Atividade física total (minutos/semana) & $248,4(335,6)$ & $187,0(188,7)$ & $253,4(372,7)$ & $189,9(198,1)$ & 0,695 & 0,886 \\
\hline
\end{tabular}

Nota: análises ajustadas por idade.

para uma visão mais integrada, ampla e prazerosa da prática de atividade física, bem como a importância do conhecimento adquirido influenciando a maneira como elas promoviam a atividade física para os usuários do SUS 21.

Algumas limitações para este estudo devem ser ressaltadas. Ao considerar o formato tradicional de ensaios clínicos, a escolha intencional e não randomizada das UBS pode ser colocada como uma das limitações metodológicas 46 . Todavia, essa escolha foi importante pelas condições favoráveis encontradas pela UBS em que foi desenvolvida a intervenção, o que facilitou o processo, fato que é muito importante em intervenções como esta que são testadas no cenário real dos serviços de saúde. Nesse sentido, alguns autores argumentam que os ensaios clínicos randomizados podem gerar muitos problemas quando tentam ser reproduzidos na prática dos serviços 47 . Já quanto ao processo de seleção amostral, ainda que tenham sido excluídas as famílias que já faziam parte de outras intervenções da pesquisa maior, não foi possível garantir que realmente não tenha havido nenhuma contaminação ou influência por conta da proximidade da vizinhança, parentescos ou pelo fato de as ACS residirem no mesmo território. Outra limitação é que não foi controlada a quantidade de visitas realizadas pelas ACS. O único dado para esse registro foi o relato do morador, portanto, é preciso considerar o viés de memória dos entrevistados. Além disso, 44,3\% dos adultos que compunham a amostra do grupo intervenção referiram não ter recebido nenhuma visita domiciliar da ACS nos últimos seis meses, logo, esse fator deve ser avaliado como uma possível limitação para o sucesso da intervenção. Outra limitação a ser considerada é a avaliação da atividade física por meio de questionários. Não foi aplicado nenhum método de avaliação direta da atividade física como o uso de acelerômetros, que fornecem avaliações mais precisas da prática de atividade física e são importantes para avaliar resultados de estudos de intervenção, podendo evitar possíveis vieses de respostas 48 . Por outro lado, a prevalência de atividade física encontrada nos adultos que participaram deste estudo é muito similar ao inquérito epidemiológico realizado com adultos daquela mesma região ${ }^{49}$, e os questionários utilizados foram validados em amostra de pessoas adultas da mesma região onde foi feita a pesquisa 30 .

A relevância do presente artigo se evidencia pela forma como a atuação profissional dos ACS vem sendo instituída: são trabalhadores na 
"linha de frente" das ações de saúde visando à transformação das condições e modos do viver, promovendo a relação e a aproximação da comunidade aos serviços de saúde e lidando com questões relativas à cidadania, à política, às relações sociais e à organização dos grupos 38,50. Dessa forma, é possível compreender que o trabalho dos ACS é complexo e transcende o campo da saúde.

Considerando a visita domiciliar como principal instrumento de trabalho do ACS no Brasil, a promoção da atividade física realizada por esses profissionais nos espaços extraunidade possui como vantagens a disponibilidade de mais tempo para o aconselhamento do que quando realizada nas UBS, permitindo regras de condutas mais flexíveis e descontraídas, e favorecendo uma abertura maior por parte dos usuários, além do ambiente domiciliar propiciar o vínculo e um melhor relacionamento com o profissional de saúde, tornando o atendimento mais humanizado.

\section{Conclusão}

Não foram encontradas evidências de efetividade de mudanças no nível de atividade física e nos estágios de mudança de comportamento em usuários do SUS/ESF que receberam visita domiciliar das ACS que passaram por um processo de formação em educação profissional para promoção da atividade física. É possível que a extrapolação para a população dos saberes adquiridos pelas ACS no processo educativo 21 não tenha ocorrido em razão das questões de processos de trabalho, limitações institucionais e demandas de outras ordens trazidas pela população, além das relações multifatoriais que permeiam a prática de atividade física. Entretanto, sugere-se que a promoção da atividade física por meio das visitas domiciliares seja discutida de forma mais ampla, como, por exemplo, pelo Ministério da Saúde e por estados e municípios; e também seja avaliada em diferentes contextos e realidades, dado que as visitas domiciliares se apresentam como mais uma possibilidade importante para o cuidado em saúde. 


\section{Resumen}

Este estudio verificó la efectividad de la promoción de actividad física realizada por agentes comunitarios de salud (ACS) en visitas a domicilios. Se trata de un ensayo controlado no randomizado que duró seis meses, con un grupo de ACS que se sometió a un proceso de formación en educación profesional para promover la actividad física en visitas a domicilios de usuarios del Sistema Único de Salud brasileño (SUS) y un grupo control. Fueron evaluadas la práctica de actividad física y las etapas de cambio de comportamiento en 176 adultos ( $n=90$ del grupo intervención y $n=86 \mathrm{del}$ grupo control) atendidos por los ACS. Se realizaron análisis de asociación, razones de prevalencia y ecuaciones de estimativas generalizadas para verificar diferencias entre los grupos. No se observaron evidencias de diferencias en los niveles de actividad física y en las etapas de cambio de comportamiento entre los usuarios de los dos grupos. Los ACS del grupo intervención realizaron más visitas a domicilios para promover actividad física a personas mayores, con bajo nivel de escolaridad, que no trabajaban y que tenían enfermedades crónicas. Es importante que los modos de trabajo y prioridades de los ACS sean reconsiderados para la ampliación de la promoción de actividad física en el SUS.

Agentes Comunitarios de Salud; Visita Domiciliaria; Actividad Motora

\section{Colaboradores}

E. F. Costa participou da elaboração dos instrumentos utilizados no estudo, coordenou o trabalho de campo, análise de dados, interpretação, elaboração de tabelas e redação final do artigo. D. R. Andrade participou da elaboração dos instrumentos utilizados no estudo, contribuiu com o trabalho de campo e colaborou na revisão do manuscrito. L. M. T. Garcia colaborou com as análises dos dados, interpretação e elaboração de tabelas e revisão do manuscrito. E. H. C. Ribeiro colaborou com a análise dos dados e revisão do manuscrito. T. I. Santos colaborou com o trabalho de campo e contribuiu com a revisão do manuscrito. A. A. Florindo elaborou o projeto, revisou e colaborou com o trabalho desde a concepção até a redação final.

\section{Agradecimentos}

À FAPESP (Fundação de Amparo à Pesquisa do Estado de São Paulo) pelo apoio financeiro (processo no 2009/14119-4), aos profissionais de saúde das UBS e à Coordenadoria Regional de Saúde Leste/Supervisão Técnica de Saúde de Ermelino Matarazzo pela colaboração.

\section{Referências}

1. Merhy EE. Um ensaio sobre o médico e suas valises tecnológicas: contribuições para compreender as reestruturações produtivas do setor saúde. Interface Comun Saúde Educ 2000; 4:109-16.

2. Ceccim R, Machado N. Contato domiciliar em saúde coletiva. Porto Alegre: Universidade Federal do Rio Grande do Sul; s.d.

3. Mattos T. Visita domiciliar. In: Kawamoto E, Santos MCH, Mattos TM, organizadores. Enfermagem comunitária. São Paulo: Editora Pedagógica e Universitária; 1995. p. 35-9.
4. Departamento de Atenção Básica, Secretaria de Atenção à Saúde, Ministério da Saúde. Política Nacional de Atenção Básica. Brasília: Ministério da Saúde; 2012.

5. Ministério da Saúde. Perfil de competências do agente comunitário de saúde. Brasília: Ministério da Saúde; 2004. 
6. Vidal SA, Silva EV, Oliveira MG, Siqueira AM, Felisberto E, Samico I, et al. Avaliação da aplicação da estratégia da Atenção Integrada às Doenças Prevalentes da Infância (AIDPI) por Agentes Comunitários de Saúde. Rev Bras Saúde Matern Infant 2003; 3:205-13.

7. Fabri CA, Waidman MAP. Visita domiciliar: a atividade do agente comunitário de saúde no programa saúde da família. Família, Saúde e Desenvolvimento 2006; 4:106-18.

8. Azeredo CM, Cotta RMM, Schott M, Maia TM, Marques ES. Avaliação das condições de habitação e saneamento: a importância da visita domiciliar no contexto do Programa de Saúde da Família. Ciênc Saúde Coletiva 2007; 12:743-53.

9. Cesar JA, Mendoza-Sassi RA, Ulmi EF, Dall'Agnol MM, Neumann NA. Diferentes estratégias de visita domiciliar e seus efeitos sobre a assistência prénatal no extremo Sul do Brasil. Cad Saúde Pública 2008; 24:2614-22.

10. Cueva M, Hicks T, Kuhnley R, Cueva K. A wellness course for community health workers in Alaska: "wellness lives in the heart of the community". Int J Circumpolar Health 2012; 71:19125.

11. Balcázar H, Wise S, Rosenthal EL, Ochoa C, Duarte-Gardea M, Rodriguez J, et al. An ecological model using promotores de salud to prevent cardiovascular disease on the US-Mexico border: The HEART Project. Prev Chronic Dis 2012; 9:E35.

12. Sranacharoenpong K, Hanning RM. Diabetes prevention education program for community health care workers in Thailand. J Community Health 2012; 37:610-8.

13. Cené CW, Haymore LB, Ellis D, Whitaker S, Henderson S, Lin FC, et al. Implementation of the power to prevent diabetes prevention educational curriculum into rural African American communities: a feasibility study. Diabetes Educ 2013; 39:776-85.

14. Teufel-Shone NI, Drummond R, Rawiel U. Developing and adapting a family-based diabetes program at the US-Mexico Border. Prev Chronic Dis 2005; 2:1-9.

15. Islam NS, Wyatt LC, Patel SD, Shapiro E, Tandon SD, Mukherji BR, et al. Evaluation of a community health worker pilot intervention to improve diabetes management in Bangladeshi immigrants with type 2 diabetes in New York City. Diabetes Educ 2013; 39:478-93.

16. Gomes MA, Duarte MFS. Efetividade de uma intervenção de atividade física em adultos atendidos pela estratégia saúde da família: Programa Ação e Saúde Floripa-Brasil. Rev Bras Ativ Fís Saúde 2012; 13:44-56.

17. Hallal PC, Knuth AG, Reis RS, Rombaldi AJ, Malta DC, Iser BPM, et al. Time trends of physical activity in Brazil (2006-2009). Rev Bras Epidemiol 2011; 14:53-60.

18. Florindo AA, Hallal PC, Moura EC, Malta DC. Practice of physical activities and associated factors in adults, Brazil, 2006. Rev Saúde Pública 2009; 43:65-73.

19. Malta DC, da Silva JB. Policies to promote physical activity in Brazil. Lancet 2012; 380:195-6.
20. Florindo AA, Mielke GI, de Oliveira Gomes GA, Ramos LR, Bracco MM, Parra DC, et al. Physical activity counseling in primary health care in Brazil: a national study on prevalence and associated factors. BMC Public Health 2013; 13:1-10.

21. Florindo AA, Costa EF, Sá TH, Santos TI, Verlardi M, Andrade DR. Physical activity promotion in primary health care in Brazil: a couseling model applied to community health workers. J Phys Act Health 2014; 11:1531-9.

22. Andrade DR, Costa EF, Ribeiro EHC, Salvador EP, Garcia LMT, Florindo AA. Do diagnóstico à ação: a experiência da pesquisa Ambiente Ativo na promoção da atividade física em Ermelino Matarazzo, na zona leste de São Paulo, SP. Rev Bras Ativ Fís Saúde 2013; 17:235-8.

23. Sallis JF, Cervero RB, Ascher W, Henderson KA, Kraft MK, Kerr J. An ecological approach to creating active living communities. Annu Rev Public Health 2006; 27:297-322.

24. Prochaska JO, Marcus BH. The transtheoretical model: applications to exercise. In: Dishman RK, editor. Advances in exercise adherence. Champaign: Human Kinetics; 1994. p. 181-90.

25. Departamento de Gestão da Educação em Saúde, Secretaria de Gestão do Trabalho e da Educação na Saúde, Ministério da Saúde. Política Nacional de Educação Permanente em Saúde. Brasília: Ministério da Saúde; 2006. (Textos Básicos de Saúde, Série Pactos pela Saúde).

26. Iñiguez L. Manual de análise do discurso em ciências sociais. Petrópolis: Editora Vozes; 2004.

27. Secretaria Executiva, Ministério da Saúde. Programa agentes comunitários de saúde (PACS). Brasília: Ministério da Saúde; 2001.

28. Florindo AA, Salvador EP, Reis RS, Guimarães VV. Percepção do ambiente e prática de atividade física em adultos residentes em região de baixo nível socioeconômico. Rev Saúde Pública 2011; 45:302-10

29. Florindo AA, Guimaraes VV, Galvao Cesar CL, de Azevedo Barros MB, Goi Porto Alves MC, Goldbaum M. Epidemiology of leisure, transportation, occupational, and household physical activity: prevalence and associated factors. J Phys Act Health 2009; 6:625.

30. Garcia LMT, Osti RFI, Ribeiro EHC, Florindo AA. Validação de dois questionários para a avaliação da atividade física em adultos. Rev Bras Ativ Fís Saúde 2013; 18:317.

31. Duggan C, Carosso E, Mariscal N, Islas I, Ibarra G, Holte S, et al. Diabetes prevention in Hispanics: report from a randomized controlled trial. Prev Chronic Dis 2014; 11:E28.

32. Gibbons MC, Tyus NC. Systematic review of USbased randomized controlled trials using community health workers. Prog Community Health Partnersh 2007; 1:371-81.

33. Carrasquillo O, Patberg E, Alonzo Y, Li H, Kenya S. Rationale and design of the Miami Healthy Heart Initiative: a randomized controlled study of a community health worker intervention among Latino patients with poorly controlled diabetes. Int J Gen Med 2014; 7:115. 
34. Islam N, Riley L, Wyatt L, Tandon SD, Tanner M, Ratnam-Mukjerji R, et al. Protocol for the DREAM Project (Diabetes Research, Education, and Action for Minorities): a randomized trial of a community health worker intervention to improve diabetic management and control among Bangladeshi adults in NYC. BMC Public Health 2014; 14:177.

35. Lopes DMQ, Beck CLC, Prestes FC, Weiller TH, Colomé JS, da Silva GM. Agentes comunitários de saúde e as vivências de prazer-sofrimento no trabalho: estudo qualitativo. Rev Esc Enferm USP 2012; 46:633-40.

36. Bauman AE, Reis RS, Sallis JF, Wells JC, Loos RJ, Martin BW. Correlates of physical activity: why are some people physically active and others not? Lancet 2012; 380:258-71.

37. Ferraz L, Aerts DRGC. O cotidiano de trabalho do agente comunitário de saúde no PSF em Porto Alegre. Ciênc Saúde Coletiva 2005; 10:347-55.

38. Tomaz JBC. O agente comunitário de saúde não deve ser um "super-herói". Interface Comun Saúde Educ 2002; 6:75-94.

39. Araújo MRN, Assunção RS. A atuação do agente comunitário de saúde na promoção da saúde e na prevenção de doenças. Rev Bras Enferm 2004; 57:19-25.

40. Brigagão J, Gonçalves R. Oficinas de promoção de saúde: discutindo os dilemas do cotidiano de um grupo de agentes comunitários de saúde. Paidéia (Ribeirão Preto) 2009; 19:387-93.

41. Theisen NIS. Agentes Comunitários de Saúde (ACS): condições de trabalho e sofrimento psíquico [Dissertação de Mestrado]. Santa Cruz do Sul: Universidade de Santa Cruz do Sul; 2004.

42. Pinafo E, Nunes E, Gonzalez A. A educação em saúde na relação usuário-trabalhador no cotidiano de equipes de saúde da família. Ciênc Saúde Coletiva 2012; 17:1825-32.
43. Oliveira DL. A "nova" saúde pública e a promoção da saúde via educação: entre a tradição e a inovação. Rev Latinoam Enferm 2005; 13:423-31.

44. Campos GWS. Um método para análise e cogestão de coletivos. 2a Ed. São Paulo: Editora Hucitec; 2005.

45. Javanparast S, Baum F, Labonte R, Sanders D, Rajabi Z, Heidari G. The experience of community health workers training in Iran: a qualitative study. BMC Health Serv Res 2012; 12:291.

46. Hulley SB, Cumming SR, Browner WS, Grady DG, Hearst NB, Newman TB. Delineando a pesquisa clínica: uma abordagem epidemiológica. Porto Alegre: Editora Artmed; 2008.

47. Victora CG, Habicht J-P, Bryce J. Evidence-based public health: moving beyond randomized trials. Am J Public Health 2004; 94:400-5.

48. Ainsworth B, Cahalin L, Buman M, Ross R. The current state of physical activity assessment tools. Prog Cardiovasc Dis 2015; 57:387-95.

49. Costa EF, Salvador EP, Guimaraes VV, Florindo AA. Atividade fisica em diferentes domínios e sua relação com a escolaridade em adultos do distrito de Ermelino Matarazzo, zona leste de São Paulo, SP. Rev Bras Ativ Fís Saúde 2010; 15:151-6.

50. Menegolla VL, Polleto DS, Krahl M. O agente comunitário de saúde no desenvolvimento de seu trabalho. Boletim da Saúde 2003; 17:81-93.

Recebido em 15/Jul/2014

Versão final reapresentada em 21/Fev/2015

Aprovado em 04/Mai/2015 